\title{
Posterior Hip Dislocation with Ipsilateral Femoral Neck Fracture: Case Report
}

Kigera J W M ${ }^{\mathbf{1}}$ MBChB, Okello J2, MBChB, MMed. 1. Department of Orthopaedics, College of Health Sciences, Makerere University, Kampala, Uganda, 2. Mulago Hospital, Kampala, Uganda Correspondence: Dr. James Kigera@yahoo.co.uk

\section{Summary}

Dislocations of the hip associated with fractures of the neck of femur are rare entities. We report a case of a 23 year old man involved in a road traffic crash and sustained a posterior hip dislocation of the right hip with an associated fracture of the neck of femur. This is a rare case but may occur in high energy injuries. He was managed by bipolar hemiarthroplasty as opposed to total hip athroplasty due to financial constrains. This case highlights the challenge of treatment of posterior hip dislocation in the setting of associated femoral neck fracture, late presentation and resource constraint.

\section{Introduction}

Dislocation of the hip associated with ipslateral fractures of the neck of femur are rare entities. (1-5). These injuries are classified as Type IV in the Stewart and Milford Classification or more comprehensively as type $3 \mathrm{~A}$ in the Brumback classification(6, 7). Usually associated with significant trauma, most patients have other concomitant injuries especially to the lower extremities(8,

9). Cases of a hip dislocation associated with a femoral neck fracture with an intact acetabulum and femoral head are extremely rare(8-10). We report a case of posterior hip dislocation with an associated fracture of the neck of the femur and an intact head of femur following a road traffic crash.

\section{Case Report}

A 23 year old patient was admitted to the emergency ward of the hospital having been referred from a peripheral rural hospital (Lira Medical Centre) following a road traffic crash 24 days prior to admission. He was a rider of a motorcycle that was involved in a head on collision with an oncoming pickup truck. He was thrown over and hit the windscreen of the pickup truck with his forehead, falling to the ground and the motorcycle falling on his right lower limb. He reported loss of consciousness for about half an hour and regained consciousness while in the hospital. He was resuscitated and diagnosed to have a fracture of the neck of femur on the right, a fracture of the distal fibula on the right, an open communited fracture of the right patella and a laceration on the forehead. The ankle fracture was immobilised, the wounds on the forehead and knee sutured after debridement and he received tetanus toxoid injection, analgesics and prophylactic antibiotics. He was subsequently referred to Mulago National Hospital.

Initial pelvic anteroposterior radiographs of the hip revealed a posterior fracture dislocation of the right hip and an intact acetabulum. (Fig. 1). Lateral Radiographs of the knee revealed a communited fracture of the patella at the inferior pole (Fig. 2 2). We also noted a stable fracture of the right ankle. (Fig. 3 ).

Total hip athroplasty was advised but due to financial constraints they could not afford to buy the implant. A decision was made to perform a hemi athroplasty with a bipolar implant. The intact femoral head was found lodged in the partly lacerated gluteal muscles and the the acetabulum was intact. The fracture neck of femur extended into the posterior part of the calcar femoris. A cementless implant was inserted and the post operative radiographs showed adequate placement. The ankle fracture was managed conservatively with a below knee plaster of paris cast while the patella injury which had undergone clinical union at the time of referral was managed by gradually increasing range of movement exercises of the knee joint. The patient was discharged non weight bearing on axillary crutches. He is currently 


\begin{tabular}{lllllll} 
Author & Number of cases & Age & Direction of Dislocation & Delay in presentation & Management & Outcome \\
\hline Sadler et al 1985(8) & 1 & 27 & Anterior & - & ORIF, Vascularised graft & Good (Healing) \\
\hline Peterson 1950(10) & 1 & 33 & Posterior & 12 Days & ORIF, Grafting & Good \\
\hline Present Study & 1 & 23 & Posterior & 24 days & Bipolar Hemiarthroplasty & Good
\end{tabular}

Table 1 Overview of literature on cases of hip dislocation associated with a femoral neck fracture and an intact acetabulum and femoral head

ambulant without any aids with no pain. He continues to be followed up.

\section{Discussion}

The combination of a posterior hip dislocation with femoral neck fracture is a rare injury. It may occur iatrogenically while attempting a closed reduction of a hip dislocation. It has also been reported to occur as part of a traumatic event as in our case $(1,2,4,5,11)$.

Posterior hip dislocations are postulated to occur after axial loading of the hip when the hip is held in flexion, adduction and internal rotation. It has also been shown that the amount of anteversion of the femoral neck differentiates those who suffer pure dislocations and those who suffer fractures of the posterior wall of the acetabu$\operatorname{lum}(12)$. It is proposed that femoral neck fractures associated with femoral head dislocations occur when the injury forces first dislocate the femoral head then force the head against the pelvis. If the energy that produced the dislocation has not been fully dissipated, the continued application of force to the leg while the femoral head abuts the pelvis will fracture the femoral neck(1). This clinical entity combined with an intact femoral head and acetabulum is rare and decision making is difficult.

Our case was managed by prosthetic replacement of the proximal femur. This was necessitated by the delay in presentation of the patients to the referral facility. Other authors have managed their cases similarly mainly due to concomitant damage to the articular surface of the femoral head(11). Sadler and DiStefano attempted open reduction and internal fixation of a basicervical femoral neck fracture associated with an anterior dislocation. The case underwent avascular necrosis and had to be salvaged by a muscle pedicle flap(8). One case we found in literature used a press fit bipolar prosthesis. This was considered to be the most conservative yet functional mode of management(2). Total hip athroplasty has been used to manage similar cases in the recent past $(9,13)$. Although about 30 cases of hip dislocation associated with a femoral neck fracture have been described(3, 14-16), many of these cases have associated fractures of the pelvis, acetabulum and the femoral head. We found only two cases in English literature that had an intact acetabulum and femoral head (Table 1). These cases have been managed by open reduction and internal fixations (ORIF). One case did not have an indication of the time period before operation but it is implied that the procedure was done emergently. The other case was done after 12 days. Our case had a long delay of 24 days and this was thought to be a poor prognostic factor precluding ORIF.

A total hip athroplasty was our preferred mode of management but was hampered by financial constraints given that the cost of a total hip replacement is five times the cost of a bipolar prosthesis. Recently, a case has been made for an algorithm to decide on preservation of the head based on the continuity of the blood supply to the femoral head(14). It would seem that this would guide surgeons in decision making. There is a case to be made for hemiarthroplasty where total joint athroplasty is not possible in cases where the vascularity is questionable. This case should alert the clinician to this rare injury and the possible modes of management available to the surgeon including the possibility of athroplasty for late presenting cases despite the intact joint surfaces. In our case the head of femur was found trapped in the gluteal muscles and it was felt that open reduction and internal fixation would not have been appropriate. Management with hemiarthroplasty will undoubtedly have long term issues that will need to be addressed including the possibility of revision in the near feature. 


\section{Posterior Hip Dislocation with Ipsilateral Femoral Neck Fracture: Case Report}

Kigera J W M, Okello J

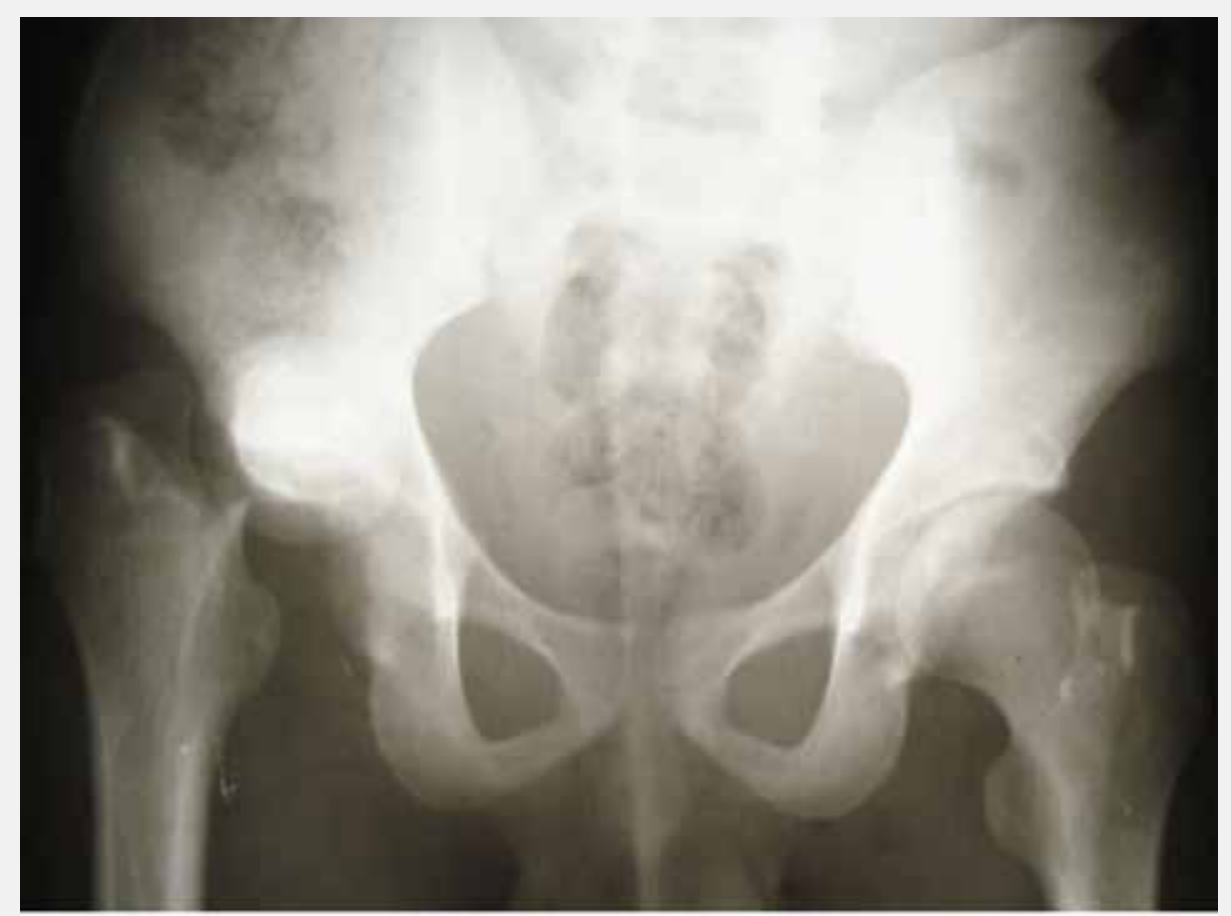

Fig. 1 AP Radiograph of the pelvis showing a posterior hip dislocation on the right with an ipslateral fracture of the neck of femur

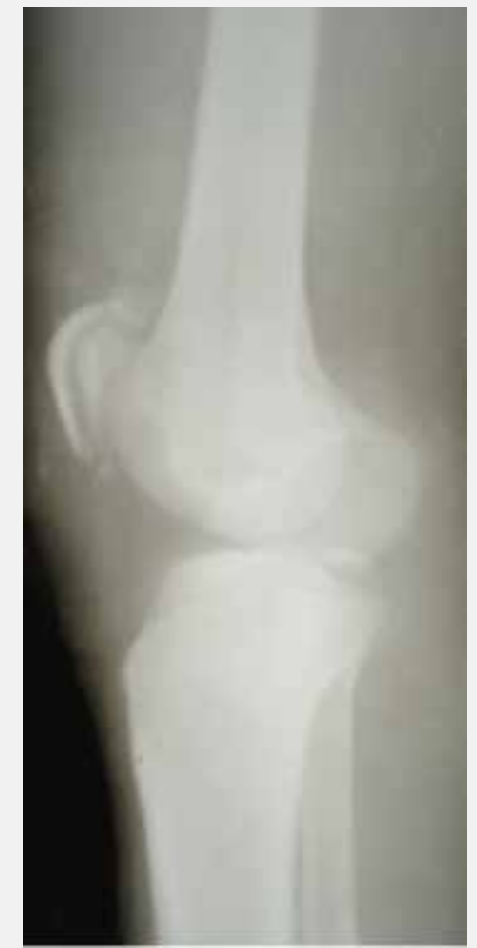

Fig. 2 Lateral Radiograph of the right knee joint showing the patella fracture

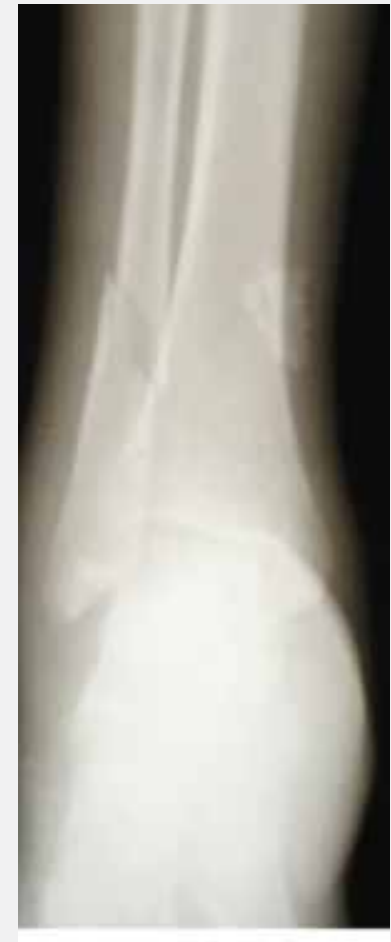

Fig. 3 AP Radiograph of the ankle joint showing the fibula fracture

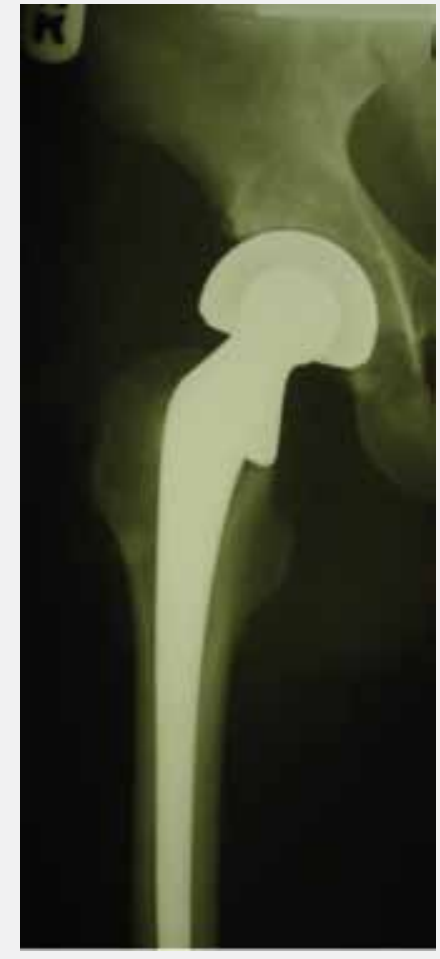

Fig. 4 Post Operative radiograph of the right hip. 


\section{References}

1. Meller Y, Tennenbaum Y, Torok G. Subcapital fracture of neck of femur with complete posterior dislocation of the hip. J Trauma. 1982;22(4):327-9.

2. McClelland SJ, Bauman PA, Mcdley CF, et al. Obturator hip dislocation with ipsilateral fractures of the femoral head and femoral neck. Clin Orth and Related Research. 1987;224:164 $-8$.

3. Mehara AK, Ramchandani GD, Sharma CS, et al. Unusual posterior hip dislocation with ipsilateral fractures of the femoral neck and head. 1995;38(4):658-9.

4. Henry AK, Bayumi M. Fractures of the femur with luxation of the ipsilateral hip. Brit J of Surg. 1934;22:204 - 30.

5. Hart VL. Fracture-dislocation of the hip. J Bone Joint Surg Am. 1942;24(2):458-60.

6. Brumback RJ, Kenzora JE, Levitt LE. Proceedings of the Hip Society, 1986, St. Louis: C.V. Mosby; 1987:181-206.

7. Stewart MJ, Milford LW. Fracture dislocation of the hip: An end result study. J of Bone and Joint Surg Am. 1954;36:315.

8. Sadler AH, DiStefano M. Anterior dislocation of the hip with ipsilateral basicervical fracture. A case report. J Bone Joint Surg Am. 1985 ;67(2):326-9.

9. Esenkaya I, Gorgec M. Traumatic anterior dislocation of the hip associated with ipsilateral femoral neck fracture: a case report. Acta Orthop Traumatol Turc. 2002;36(4):366-8.

10. Peterson LT. Dislocation of the hip associated with fracture of the neck of femur. J Bone Joint Surg Am. 1950;32:274-9.

11. Mehara AK, Das Ramchandani G, Sharma CS, et al. Unusual posterior hip dislocation with ipsilateral fractures of the femoral neck and head. J Trauma. 1995;38(4):658-9.

12. Upadhyay SS, Moulton A, Burwell RG. Biological factors predisposing to traumatic posterior dislocation of the hip. J Bone Joint Surg [Br]. 1985;67:232-6.

13. Dummer RE, Sanzana ES. Hip dislocations associated with ipsilateral femoral neck fracture. Int Orthop. 1999;23(6):3534.

14. Tannast M, Mack PW, Klaeser B, et al. Hip dislocation and femoral neck fracture: Decision-making for head preservation. Injury, Int J Care Injured. 2009;40 1118-24.

15. Hougaard K, Thomsen PB. Traumatic posterior fracture-dislocation of the hip with fracture of the femoral head or neck, or both. J Bone Joint Surg Am. 1988;70(2):233-9.

16. Polesky RE, Polesky FA. Intrapelvic dislocation of the femoral head following anterior dislocation of the hip: A case report. J Bone Joint Surg Am. 1972;54:1097-8. 